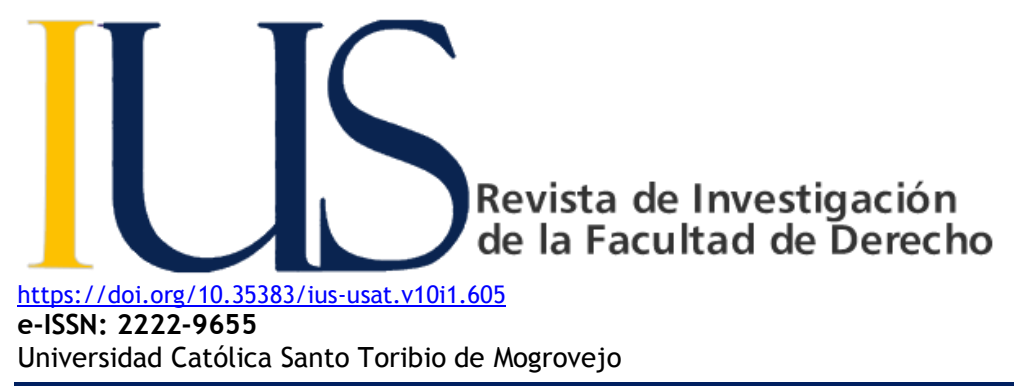

\title{
El Contrato Administrativo de Servicios, los trabajadores de los hospitales y la eventual necesidad de establecer un tratamiento jurídico diferenciado
}

\author{
The Administrative Service Contract, hospital workers and the eventual \\ need to establish a differentiated legal treatment \\ Jesús Enrique Aguinaga Saavedra *, 1, a \\ jaguinaga.sie@gmail.com \\ https://orcid.org/ 0000-0002-5004-3303 \\ ${ }^{*}$ Autor corresponsal \\ ${ }^{1}$ Universidad Católica Santo Toribio de Mogrovejo, \\ Chiclayo, Perú \\ a Abogado.
}

\section{Fechas importantes}

Recibido: 2021-05-28

Aceptado: 2021-07-19

Publicado online: 2021-07-31

\begin{abstract}
Resumen
El Contrato Administrativo de Servicios (CAS), diseñado para superar una serie de contingencias de carácter laboral en el Estado, hasta la implementación de un régimen único, es muy utilizado en el Sector Salud. Sin embargo, durante la primera ola de contagios, ocasionados por la Covid-19, ha mostrado su principal carencia: la falta de estabilidad, con el personal médico y asistencial de los hospitales del país, que son el personal más expuesto y sacrificado de la primera línea de defensa. Esto es un problema que merece pronta solución, teniendo en cuenta la demanda de inconstitucionalidad dirigida contra la Ley $n .{ }^{\circ} 31131$. En ese sentido, se propone un tratamiento jurídico que garantice la estabilidad laboral de estos trabajadores, como justo reconocimiento a su enorme sacrificio, teniendo en cuenta la Constitución Política del Perú, los criterios del Tribunal Constitucional y la legislación vigente.
\end{abstract}

Palabras clave: igualdad; tratamiento diferenciado; Contrato administrativo de servicios.

\section{Abstract}

The Administrative Services Contract (CAS), designed to overcome a series of labor contingencies in the State, until the implementation of a single regime, is widely used in the Health Sector. However, during the first wave of infections, caused by Covid-19, it has shown its main deficiency: the lack of stability, with the medical and assistance personnel of the country's hospitals, which are the most exposed and sacrificed personnel of the first line of defense. This is a problem that deserves prompt solution, taking 
into account the unconstitutionality claim directed against Law N. ${ }^{\circ} 31131$. In this sense, a legal treatment is proposed that guarantees the job stability of these workers, as fair recognition of their enormous sacrifice, taking into account the Political Constitution of Peru, the criteria of the Constitutional Court and current legislation.

Keywords: equality; differentiated treatment; Administrative service contrac

\section{Introducción: Igualdad y diferencia como consideraciones Previas}

\section{Igualdad}

La igualdad revela correspondencia o conformidad en determinados sujetos (u objetos), partes o aspectos de estos, o equivalencia. Es una abstracción, por sí sola no tiene existencia; sin embargo, influye en toda la sociedad.

\section{Igualdad en la formación del derecho}

La igualdad es un concepto que ha tenido una influencia importante en los grupos humanos. Podemos entender a la sociedad moderna como un grupo de culturas que, históricamente, son producto de un largo devenir de muchos grupos humanos que -unos a otros- se eliminaron progresivamente para obtener cierto control de su destino colectivo. Todo lo anterior con miras a la estabilidad y supervivencia de su identidad, con todas las condiciones favorables que dicho logro implicaba (libertad, mayores recursos para subsistir, mayor posibilidad de riqueza, entre otros).

¿Cómo se formaron esos grupos?, ¿cómo se enfrentaron? Igualdad y diferencia son las claves. El hombre no ha sabido distinguir que tan importantes o relevantes son sus coincidencias, y en ese sentido, hubo quienes que se creyeron mejores que otros ${ }^{1}$. Es con el descubrimiento de la igualdad natural ${ }^{2}$ (que parte de la dignidad humana) que verdaderamente se forma un concepto sólido de igualdad, libertad y justicia; y, con la revolución liberal, iniciada en Francia, que se modifica radicalmente una serie de concepciones, consagrando a la igualdad como uno de los derechos fundamentales del hombre y del ciudadano ${ }^{3}$. Así lo expresan varias normas internacionales, como, por ejemplo, el artículo 6 de la Declaración de los Derechos del Hombre y del Ciudadano de 1789: «La ley es la expresión de la voluntad general (...) Debe ser la misma para todos, tanto si protege como si Castiga. Todos los ciudadanos, al ser iguales ante ella, son igualmente admisibles a todas las dignidades, puestos y empleos públicos, según su capacidad y sin otra distinción que la de sus virtudes y la de sus talentos».

\section{Diferencia}

Significa algo que se distingue, que no concuerda, algo que no se reproduce exacta o relativamente igual que determinados sujetos (u objetos), partes o aspectos de estos; 0 , simplemente, algo que no es equivalente.

La diferencia también es una abstracción. Para determinarla se puede formular las preguntas básicas que Bobbio (1993, p. 54) usaba con la igualdad: ¿entre quienes? y ¿en qué?

\footnotetext{
${ }^{1}$ Lógicamente hubo muchos otros factores que determinaron la aniquilación entre hombres, y que aún lo hacen; sin embargo, por orden metodológico, sólo trabajamos sobre la igualdad.

2 Por eso el hombre se agrupa para sobrevivir, pero enseguida busca conquistar a otros hombres y tratarlos de esclavos, y cuando la propia aniquilación de su grupo se detiene, entonces busca explotar a otros hombres de otros territorios. Prohibida la aniquilación y la esclavitud, la explotación comienza en manos de aquellos que tienen más recursos, como carencias de la igualdad que hasta ese entonces fue reconocida, motivo por el cual nació el Derecho del Trabajo.

${ }^{3}$ Cfr. BERNALES BALLESTEROS, E (1999). La Constitución de 1993: análisis comparado, Quinta edición, Perú: RAO, p. 114.
} 
Se mencionó que la igualdad ha sido fuente inspiradora de Derecho. No obstante, el mismo, también ha sido inspirado por las diferencias que pueden estar reflejadas, bien para distinguir o bien para igualar (como lo veremos posteriormente). La diferencia y la igualdad son fuentes de inspiración complementarias para crear normas.

\section{Diferencias en la formación del Derecho}

Tenemos claro que las normas son creadas para reconocer igualdad y ser dirigidas a una generalidad de sujetos, como también pueden ser creadas para establecer distinciones. Por eso, nos parece importante mencionar cómo son reflejadas las diferencias en el derecho.

Estas diferencias pueden ser justificadas o no justificadas. Son justificadas, cuando dichas distinciones normativas se producen en base a la naturaleza de las cosas (art. 103 de la Constitución ${ }^{4}$ ) atendiendo singulares características o condiciones de determinados sujetos 0 grupos. Las diferencias justificadas se pueden establecer de tres maneras, en la norma (Constitucional o de cualquier otro rango) ${ }^{5}$ : la primera es reconociendo derechos, por ejemplo, la atención preferencial a los minusválidos o madres embarazadas, la protección especial para el trabajo infantil, las ventajas tributarias en aquellas zonas donde la actividad empresarial no llega; la segunda es no reconociéndolos, como en el caso de los trabajadores de la microempresa a los cuales no se les reconoce el acceso a un seguro complementario de trabajo de riesgo; y la tercera es prohibiéndolos, como la restricción que tienen los jueces o los policías para realizar una huelga ${ }^{6}$. Cuando se produce una diferencia justificada se genera una equidad. Cuando estas diferencias son no justificadas, habría que realizar una distinción adicional, es decir, dichas diferencias podrían ser desigualdad o discriminación. La primera (desigualdad) es una distinción que carece de justificación; la segunda (discriminación), en cambio, no carece de justificación, pero está basada en algún motivo proscrito (sexo, raza, creencias, orientación sexual, discapacidad, entre otras), motivos que históricamente han producido aberración y calamidad del hombre contra el hombre y que los Estados han rechazado de manera tajante y expresa.

No puede confundirse desigualdad y discriminación. Existe la afectación a un derecho si a un joven no le permiten la admisión a una universidad pública, a pesar de haber logrado una calificación de mérito que permite su ingreso, por el hecho que sus padres son acaudalados económicamente $y$, por tanto, tienen los recursos para solventar su educación en cualquier universidad privada. Sin embargo, los motivos que generan dicho acto no pueden considerarse discriminación (en el sentido que hemos explicado). Los recursos económicos de los padres no son causas proscritas, ya que no están inclusos en el grupo de causas de "otra índole" (Defensoría del Pueblo, 2007, p. 38).

\section{Igualdad y diferencia en el orden jurídico: principio de igualdad}

\footnotetext{
${ }^{4}$ Artículo 103.-

Pueden expedirse leyes especiales porque así lo exige la naturaleza de las cosas, pero no por razón de las diferencias de las personas (...)".

${ }^{5}$ Esta clasificación ha sido inspirada por las referencias a los derechos que se puede encontrar en las constituciones modernas, de acuerdo a NEVES (2014, p. 65).

${ }^{6}$ El artículo 42 de la Constitución Política del Perú reconoce la sindicación y la huelga como derechos de los trabajadores del Estado. Sin embargo, excluye a los que ostentan capacidad de decisión y a los que tienen cargos de confianza o de dirección; además los que sirven en las FFAA y la PNP.
} 
Al hablar de ordenamiento jurídico, la igualdad y la diferencia - explicadas de forma genérica - siempre se reflejarán en términos de igualdad, incluso para establecer diferencias. No se encontrará un derecho a la diferencia, pero si un derecho a la igualdad (art. 2, numeral 2 $\mathrm{CPP}^{7}$ ); y si se establecen distinciones (art. 103 CPP), las mismas tienen el fin de brindar igual trato a un grupo de iguales $y$, diferente, a un grupo de diferentes, para que a los integrantes de cada grupo se trate con igualdad, bajo sus mismas reglas.

En la actualidad, la igualdad tiene tres identidades, que le otorgan distintas funciones: es Principio, es Derecho y Valor Superior. Todas estas identidades y funciones tienen carácter constitucional. Reflexionaremos de forma breve sobre el derecho, principio y valor de la Igualdad ante la ley en su dimensión de igualdad en la creación de la ley - de evidente origen liberalista, pero que iría evolucionando con aportes de la política social - al momento de establecer diferencias.

La ley no puede asumir que todos los individuos son iguales, no puede atribuirles los mismos derechos y obligaciones, si es que se busca un bienestar público (progreso social y económico). En ese sentido, el legislador debe revisar los objetivos de las normas que pretende hacer vigentes, identificar al grupo de sujetos sobre los que pretende aplicarla y evaluar las posibles consecuencias de su aplicación. Esta evolución de la igualdad en la ley ha permitido la segmentación del derecho, el derecho al trabajo y el derecho al consumidor son dos grandes ejemplos; además, ha inspirado herramientas para combatir la desprotección social, como las medidas de discriminación positiva ${ }^{8}$.

Resulta notorio que, el Estado podría configurar distinciones que, desde un primer plano, podrían resultar no justificadas. Ante estas situaciones, considerando el fundamento jurídico 2 del fallo de nuestro Tribunal Constitucional, recaído sobre el expediente N. ${ }^{\circ}$ 00018-2003AI/TC, Gutiérrez y Sosa (2005) sostienen que el citado colegiado indica que no existen conflicto entre la igualdad ante la ley y las normas diferenciadoras, siempre que se cumpla con una serie de condiciones, tales como la concurrencia de distintos supuestos que hagan necesaria una distinción; la existencia de un fin determinado; la razonabilidad que la sostenga, la existencia de proporcionalidad - es decir: correspondencia entre los supuestos de hecho y el fin; y, finalmente, racionalidad, que supone una relación coherente de las situaciones, los medios utilizados y su finalidad.

Toda norma diferenciadora debe validar su existencia conforme al test de proporcionalidad, herramienta que sirve para analizar la constitucionalidad de las disposiciones que limitan derechos o que establecen distinciones, que en críticas de la doctrina nacional ha sido usado de forma desordenada por el Tribunal Constitucional (Rubio, 2011); y, que con la sentencia del Tribunal Constitucional N 0045-2004-PI-TC consolidó su estructura, de la siguiente forma:

i) La intervención en el principio-derecho igualdad

ii) Determinación de la intensidad de la intervención

iii) El fin del tratamiento diferente

iv) Examen de la idoneidad del tratamiento diferente

v) Examen de necesidad

\footnotetext{
${ }^{7}$ La Constitución el derecho de igualdad en el inciso 2 artículo 2, el cual señala que toda persona es igual ante la ley; y, que no se debe discriminar en razón del origen, raza, sexo, idioma, religión, opinión, condición económica o de cualquier otra índole".

${ }^{8}$ Al respecto, Landa Arroyo señala que, "[a] partir de la idea de igualdad se puede entender que todo tratamiento diferencia que no esté debidamente justificado resulta discriminatorio $y$, por tanto, prohibido por el ordenamiento jurídico. Asimismo, no todo tratamiento diferenciado entre las personas estaría prohibido, ya que existirían diferencias relevantes entre ellas que harían injusto tratar de forma idéntica situaciones que, en los hechos, son diferentes. LANDA ARROYO, C. (2017). Los Derechos Fundamentales. Lima: Editorial PUCP. P. 31.
} 
vi) Examen de proporcionalidad en sentido estricto

\section{Revisión de trayectoria del Contrato administrativo de servicios en el Perú}

El régimen especial laboral de la contratación administrativa de servicios (régimen CAS) se creó en el 2008, a través del decreto legislativo n. ${ }^{\circ} 1057$. Este régimen especial de la Administración pública fue instituido por las siguientes razones:

a) Detener los contratos de servicios no personales, que hasta ese entonces era un sistema de contratación en la que el Estado reconocía abiertamente que el servidor realizaba labores subordinadas y permanentes, sin estar vinculado laboralmente con la entidad. Es decir, se trabaja sin tener ninguna herramienta de protección o beneficios laborales que el marco jurídico señalaba.

b) Prohibir que las instituciones del Sector Público utilicen este tipo de contrato para los puestos de naturaleza permanente, por medio de las empresas de servicios especiales, o temporales, o de cooperativas de trabajadores.

c) Evitar las incorporaciones, que el Poder Judicial disponía a los servidores en el régimen de los Decretos Legislativo 276 y 728.

La Ley . $^{\circ} 29849$ (2012) señala que este régimen laboral tendrá fin; sin embargo, no establece una fecha límite, cuando la intención de varios legisladores era poner un límite claro y preciso ${ }^{9}$. La primera disposición complementaria de esta norma solo anuncia la extinción progresiva del régimen CAS, desde el año 2013, con la implementación del Régimen del Servicio Civil.

Además del anuncio de la eliminación del CAS, la norma referida realiza algunas mejoras en los derechos de este régimen:

a) Remuneración no menor al mínimo vital.

b) El aumento de vacaciones a 30 días.

c) Acceder a los derechos que reconoce la Ley de Seguridad y Salud en el Trabajo ${ }^{10}$.

d) Un lapso para el refrigerio, que no integra la jornada laboral.

e) Aguinaldo de fiestas patrias y navidad, según la ley.

Estas mejoras se sumaron a las realizadas con el Decreto Supremo n. ${ }^{\circ}$ 65-2021-PCM, expedido por consecuencia de la invocación que se realiza la Sentencia del Tribunal Constitucional recaída sobre el Expediente $\mathrm{N}^{\circ}$ 002-2010-PI/TC, por las que se incluyó los derechos de negociación colectiva y huelga.

Aún con estos refuerzos, el régimen CAS genera un problema fundamental: la inestabilidad laboral, con independencia del rendimiento del trabajador. Ello, en vista de que el artículo 5 del D. Leg. $n .{ }^{\circ} 1057$ señala que se celebra a plazo determinado y es renovable; y, que, el artículo 5 del Reglamento de la citada norma, aprobado por Decreto Supremo n. ${ }^{\circ}$ 075-2008-

\footnotetext{
${ }^{9} \mathrm{En}$ el presente enlace podrá descargarse el referido diario de debates, en relación a la Ley n. ${ }^{\circ} 29849:$ http://spij.minjus.gob.pe/Graficos/Peru/2012/Abril/06/L-29849.pdf

${ }^{10}$ Se recomienda revisar el D. leg. $n .^{\circ} 1057$ y, además, la Ley n. ${ }^{\circ} 29849$.
} 
PCM, indica que la renovación depende de la voluntad de la entidad ${ }^{11}$. Esta contingencia fue advertida por el pleno ${ }^{12}$.

Esta particularidad, empobrece las condiciones de trabajo de muchos servidores, que para mantenerse vigentes en sus puestos, deben aceptar condiciones más severas de trabajo, que aquellos contratados por los regímenes laborales del Decreto Legislativo $n .^{\circ} 276$ y del Decreto Legislativo n. ${ }^{\circ} 728$ o el régimen del servicio civil. Una jornada más extensa de trabajo, poco respeto a la desconexión digital, trabajos en momentos de descanso establecidos en la norma, son algunos ejemplos de lo que los servidores CAS se ven obligados a tolerar.

El informe del régimen CAS, del año 2017, elaborado por la Autoridad Nacional del Servicio Civil - Servir ${ }^{13}$, señala que los servidores con contrato administrativo de servicios constituyen el segundo grupo más numeroso del empleo público, después de los servidores sujetos a regímenes de carreras especiales, en tanto representan al $22 \%$ de la masa laboral estatal.

\section{EI CAS en los hospitales del País}

Los índices expuestos en el apartado anterior se reflejan también en las instituciones del Sector Salud. Según se advierte del dictamen del proyecto de ley, que fue insumo de la Ley n. 31039 - que, entre otras cosas, dispuso el nombramiento de los trabajadores de la salud contratados por el régimen CAS - en los hospitales del Ministerio de Salud, la contratación administrativa de servicios es el segundo régimen más empleado después del régimen laboral del decreto legislativo 276, con un total de 54143 servidores. De acuerdo con una declaración del Sr. Omar Merino López, entonces Presidente de la Comisión de Salud, existe un aproximado de 23320 trabajadores, que incluyen profesionales, cirujanos, auxiliares asistenciales y técnicos contratados con este régimen ${ }^{14}$.

En el Seguro Social de Salud (Essalud), la utilización del CAS no era tan elevada. De acuerdo con el Proyecto de Ley . $^{\circ} 322 / 2016-\mathrm{CR}^{15}$, el CAS ocupa el tercer lugar como modalidad de contratación. A diciembre de 2016, había 11917 servidores contratados con dicho régimen. Con la emisión de la Ley $n$. $^{\circ} 30555$, se dispone el traslado de profesionales de la salud, técnicos y auxiliares asistenciales de la salud, profesionales administrativos; y, técnicos y auxiliares administrativos, que hayan ingresado mediante concurso y que hayan laborado de forma continua por más de 2 años. Dicha incorporación no es inmediata, tiene un plazo máximo de 3 años, y atiende las necesidades de Essalud, además de las disposiciones del reglamento de dicha norma - todo ello según el artículo 4 de la citada ley. No obstante, la entidad ha seguido convocatorias con dicho régimen.

\footnotetext{
${ }^{11}$ Artículo 5.- Duración del contrato administrativo de servicios

5.1. El contrato administrativo de servicios es de plazo determinado. La duración del contrato no puede ser mayor al período que corresponde al año fiscal respectivo dentro del cual se efectúa la contratación; sin embargo, el contrato puede ser prorrogado o renovado cuantas veces considere la entidad contratante en función de sus necesidades. Cada prórroga o renovación no puede exceder del año fiscal y debe formalizarse por escrito antes del vencimiento del plazo del contrato o de la prórroga o renovación anterior.

12 El congresista Hurtado Zamudio señaló que: «[...] ya están experimentando el famoso mobbing, un acoso por el cual a una persona que trabaja de la misma manera se le paga diferente. Eso es lo que está pasando en el sector estatal: una persona trabaja y hace lo mismo sin que se le concedan los mismos beneficios sociales ni igual trato»

${ }^{13}$ Ente rector en el sistema administrativo de gestión de recursos humanos del Estado

14 ALARCÓN, Luz (26 de agosto de 2020). Unos 23000 trabajadores CAS pasarán al régimen 276. La República. https://larepublica.pe/economia/2020/08/26/unos-23000-Cas-del-sector-salud-pasaran-al-regimen-276/?ref=Ire
}

${ }^{15}$ Este proyecto sirvió de insumo para la Ley n. ${ }^{\circ} 30555$, Ley que incorpora al Régimen Laboral del Decreto Legislativo No 728 a los trabajadores profesionales, no profesionales, asistenciales y administrativos de ESSALUD que se encuentran bajo el Régimen de Contratación Administrativa de Servicios. 


\section{Covid-19, y la precariedad de los CAS de la primera línea en el Sector Salud}

En marzo del 2020, la Organización Mundial de la Salud (OMS) determinó que el estado de contagio masivo a nivel internacional, generada por el virus Covid-19, constituye una pandemia, puesto a que en más de 100 naciones se reportó contagios.

La aparición de esta enfermedad ocasionó que se declare estado de emergencia sanitaria nacional, mediante Decreto Supremo n. ${ }^{\circ}$ 008-2020-SA, con el fin de establecer medidas para la prevención y control de la enfermedad. Dicho estado de emergencia ha sido prorrogado por los decretos supremos n. ${ }^{\circ} 020-2020-S A, n{ }^{\circ} 027-2020-S A, n .{ }^{\circ} 031-2020-S A$ y $n .{ }^{\circ} 009-2021-$ SA, hasta el 02 de setiembre de 2021. Además, se establecieron medidas restrictivas a los derechos individuales, tales como la cuarentena, cierre de fronteras, restricción de actividades económicas, toques de queda, entre otras ${ }^{16}$.

De acuerdo con la data ofrecida por la Universidad Johns Hopkins ${ }^{17}$, en Perú se contabiliza un total de 1889052 Casos de coronavirus, con 66220 muertes. Dicha información es similar a la mostrada por el Ministerio de Salud, que determina 1884596 contagios y 65911 muertes $^{18}$.

Como se sabe, el personal que brinda servicios en los hospitales, resultó exento de las restricciones de tránsito, por mérito del literal c) de párrafo 4.1. del artículo del Decreto Supremo n. ${ }^{\circ}$ 44-2020-PCM ${ }^{19}$, por constituir parte de la primera línea de contención de la pandemia en el país. Este grupo de trabajadores, por estar prestando servicios en contacto directo con los portadores de la enfermedad, y por la falta de gestión de las entidades competentes, se ha visto expuesto a las siguientes problemáticas:

a) Falta de equipo de protección personal ${ }^{20}$; ocasionando el reclamo del decano del Colegio Médico del Perú, entre otros representantes del personal de salud.

b) 121 enfermeras han muerto desde que empezó la pandemia, 32 en el 2021; 42 están en la unidad de cuidados intensivos; 74, hospitalizadas; y, 267 en aislamiento domiciliario por COVID-19, cifra que probablemente aumente durante la publicación de este trabajo. Ello, de acuerdo con las declaraciones de la Decana del Colegio de Enfermeras del Perú, Mg. Liliana La Rosa Huertas ${ }^{21}$.

\footnotetext{
${ }^{16}$ Lo referido puede obtenerse de la revisión de algunas normas, entre las que figuran: Decreto Supremo N. ${ }^{\circ}$ 044-2020-PCM; Decreto Supremo N. ${ }^{\circ} 184-2020-P_{C M}$, modificado por el Decreto Supremo N. ${ }^{\circ} 002-2021-P C M$, el Decreto Supremo N. ${ }^{\circ} 008-2021-P C M$ y el Decreto Supremo N ${ }^{\circ} 036-$ 2021-PCM.

${ }^{17}$ Coronavirus Resource Center (2021). Tablero COVID-19 por el Centro de Ciencia e Ingeniería de Sistemas (CSSE) de la Universidad Johns Hopkins (JHU). https://coronavirus.jhu.edu/map.html.

${ }^{18}$ Sala Situacional Covid-19 Perú. Muertes y contagios a nivel nacional. https://covid19.minsa.gob.pe/sala_situacional.asp.

19 "Artículo 4.- Limitación al ejercicio del derecho a la libertad de tránsito de las personas4.1 Durante la vigencia del Estado de Emergencia Nacional y la cuarentena, las personas únicamente pueden circular por las vías de uso público para la prestación y acceso a los siguientes servicios y bienes esenciales:

(...)

c) Asistencia a centros, servicios y establecimientos de salud, así como centros de diagnóstico, en Casos de emergencias y urgencias. $(\ldots)^{\prime \prime}$.

${ }^{20}$ En el Hospital Rebagliati (Jesús María) el Dr. Teodoro Quiñones, director del Sindicato Nacional Médico del Seguro Social (Sinamssop), señaló a Canal $\mathrm{N}$ que existe una considerable grupo de personal de la salud que se ha visto perjudicado por la fala de equipos de protección. Ello ha provocado que se realicen protestas en varios hospitales de Essalud, a lo largo del país. Sociedad La República (18 de junio de 2020). Coronavirus en Perú: personal de EsSalud protestó por falta de equipos de protección. La República. https://larepublica.pe/sociedad/2020/06/18/coronavirus-en-perupersonal-de-essalud-protesto-por-falta-de-equipos-de-proteccion-mdga/
}

21 Diario Correo (7 de marzo de 2021). 121 enfermeras han fallecido por la COVID-19 desde el inicio de la pandemia. Diario Correo. https://diariocorreo.pe/peru/coronavirus-peru-121-enfermeras-han-fallecido-covid-19-desde-inicio-pandemia-noticia/?ref=dcr. 
c) Según el Colegio Médico del Perú, 366 médicos fallecieron debido a la COVID-19. Actualmente, hay 13.073 galenos contagiados en todo el país ${ }^{22}$, cifras que probablemente aumente durante la publicación de este trabajo.

A pesar de haber perdido colegas, amigos, familiares; $y$, a pesar de trabajar varias horas sin descanso, pese a que - en muchos casos - las remuneraciones se atrasan por meses y se carece de implementos médicos y de seguridad suficientes, que son suplidos con el peculio de los propios trabajadores; y, pesar de que día a día el personal de salud viene arriesgando su vida, en dos últimos meses del año 2020, cuando la primera ola de la pandemia estaba terminando y, el contexto se veía dotado de un poco de tranquilidad, se comenzaron a emitir cartas de no renovación del contrato a muchos trabajadores que habían ingresado por concurso público y que tenían varios años de servicios en los hospitales ${ }^{23}$. Es decir, se prescindió de profesionales que no sólo ingresaron compitiendo públicamente en el marco de un concurso de méritos, sino, además, que durante varios años han venido convalidando su permanencia a base de trabajo y esfuerzo - no tendría sentido, sobre todo antes de la pandemia, pensar que un profesional no es un buen elemento después de ser renovado, por hasta más de 9 años.

\section{Reacciones normativas ante la Contratación Administrativa de Servicios}

La inestabilidad que genera el CAS a los trabajadores del Estado peruano ha provocado que se formulen diversas propuestas legislativas dirigidas a eliminar este régimen laboral. De esta manera, en el año 2020 se publicó la Ley n. 31039 que, entre otras cosas, dispuso el nombramiento de los profesionales, técnicos y auxiliares asistenciales de la salud contratados por el Ministerio de Salud, mediante la contratación administrativa de servicios. El Tribunal Constitucional declaró su inconstitucionalidad, con sentencia recaída en el expediente Exp. $\mathrm{N}^{\circ}$ 00011-2020-PI/TC. Este fallo guarda correspondencia con el ordenamiento jurídico vigente, entre otras razones, porque la norma derogada mantenía los tratamientos diferenciados entre trabajadores, problema que se intenta superar con la implementación del régimen del servicio civil.

Posteriormente, se aprobó la Ley $\mathrm{n}^{\circ} 31131$, Ley que establece disposiciones para erradicar la discriminación en los regímenes laborales del Sector Público. Entre otras cosas, esta norma dispone el traslado del régimen laboral regulado por el Decreto Legislativo n. ${ }^{\circ} 1057$ (régimen CAS) a los regímenes laborales regulados por el los decretos legislativos $n .^{\circ} 276$ y n. ${ }^{\circ} 728$, de acuerdo a la entidad; la paralización de la contratación regulada por el régimen CAS y estabilidad de aquellos servidores regulados por el régimen CAS hasta que se incorporen a los regímenes señalados ${ }^{24}$, condición indeterminada que es ratificada por el Informe n. ${ }^{\circ}$ 357-2021SERVIR/GPGSC.

El 13 de mayo de este año, el Tribunal Constitucional admitió a trámite, por unanimidad, la demanda de inconstitucionalidad contra la Ley $n .^{\circ} 31131$. Teniendo en cuenta que esta norma

\footnotetext{
22 Sociedad La República (2 de marzo de 2021). Coronavirus: 366 médicos fallecieron y 50 permanecen en UCI, reporta el CMP. La República. https://larepublica.pe/sociedad/2021/03/03/coronavirus-366-medicos-fallecieron-y-50-permanecen-en-uci-reporta-el-cmp/?ref=Ire.

23 Debe señalarse que por la Única Disposición Complementaria Final del Decreto de Urgencia n. ${ }^{\circ} 086-2020$, se contrató personal con el régimen Cas, liberándose a las entidades de realizar un concurso público, requisito indicado en el artículo 8 de la norma citada. Estos contratos son temporales y se resuelven una vez que termine el estado de emergencia sanitaria.

${ }^{24}$ La emisión de esta norma generó un gran impacto en la mecánica de contrataciones del Estado, al punto que todos los concursos CAS para funciones permanentes se suspendieron. No obstante, el 31.3.2021, se publicó el Decreto de Urgencia n. ${ }^{\circ}$ 34-2021, cuya segunda disposición complementaria final rompe las reglas de la Ley $n .^{\circ} 31131$, pues permite que las entidades puedan realizar concursos CAS sin distinguir la temporalidad de la necesidad; modifica algunas características del proceso de convocatorias para que tenga mayor velocidad; y, ajusta todo este periodo de contrataciones a fechas límites: 17.3.2021 para las convocatorias y 31.12.2021 para la vigencia de los contratos.
} 
comparte una lógica muy similar a la de la Ley $n .^{\circ} 31039$, es muy probable que el TC declare su inconstitucionalidad. Asimismo, existe la probabilidad de que los trabajadores amparados por los efectos de la Ley $n .^{\circ} 31131$ pierdan la condición indeterminada en sus contrataciones - aunque ello sea materia de cuestionamiento ${ }^{25}$.

El escenario ocurrido en los últimos meses del año 2020 podría retornar; y, pasada la segunda ola de contagios, o terminada la pandemia, varios profesionales que han arriesgado su vida, en condiciones de trabajo deplorables, durante la peor crisis sanitaria del país, y que se encuentran bajo un régimen laboral cuya desaparición ha sido postergada injustificadamente por el Estado, podrían ser despedidos.

\section{Propuesta normativa para la protección de los CAS}

De ocurrir tal supuesto, corresponde presentar una propuesta de solución, dirigida únicamente a los trabajadores que presten servicios presenciales en los hospitales a nivel nacional: profesionales, técnicos, auxiliares asistenciales. La primera parte consiste en disponer el nombramiento del personal señalado en el régimen respectivo de los hospitales en los que se encuentren prestando servicios, sea bajo el régimen del Decreto Legislativo $n .^{\circ} 276$ o bajo el régimen del Decreto Legislativo $\mathrm{n}^{\circ}{ }^{728}$. La segunda parte consiste en el acceso inmediato sin concurso público - al régimen del servicio civil, cuando este se implemente en las entidades. La primera parte puede obviarse si es que el traslado efectivo al régimen del servicio civil se produce en un plazo prudencia ( 1 o 2 años $)^{26}$.

Para ello pueden establecerse una serie de condiciones, como:

A) Haber ingresado a su puesto de trabajo vía concurso público. Debe tenerse en cuenta que en el marco del estado de emergencia sanitaria se emitieron disposiciones para permitir que personal médico ingrese a trabajar a los hospitales, mediante el régimen CAS, pero exceptuándolos de pasar por el respectivo concurso público. Ello bajo la lógica de que se necesitaba personal en la primera línea. Entonces, se advierte claramente que las contrataciones descritas tienen naturaleza temporal. No sería equitativo incluirlos pues son personal que entró con claras ventajas únicamente para brindar servicios en circunstancias complicadas.

B) Tener un periodo continuo de trabajo mínimo de un (1) año seguido, para una entidad, o de dos (2) años discontinuos, en labores de naturaleza permanente. Para ello se toma como referencia los periodos mínimos en las leyes $n .^{\circ} 31039$ y n. ${ }^{\circ} 31131$. Es de suponer que el personal que tiene ese tiempo de servicios en el Estado ha demostrado su eficiencia.

C) Estar desempeñando funciones de naturaleza permanente. El objetivo es proteger a los trabajadores CAS de los hospitales que, durante años, mediante su esfuerzo han convalidado su presencia en el Sector Público; y, que se les prive del derecho a seguir trabajando, basándose en una norma cuestionable.

Mientras las incorporaciones mencionadas se ejecuten, se debe brindar estabilidad laboral a los trabajadores que cumplan con las condiciones señaladas.

\footnotetext{
${ }^{25}$ Pueden surgir varias posturas al respecto. La primera es que en tanto la Ley $n .^{\circ} 31131$ modifica el artículo 5 del Decreto Legislativo $N^{\circ} 1057$, relativo a la temporalidad del CAS, al ser declarada inconstitucional, este recuperaría su vigencia.

${ }^{26}$ Debe aclarase que se considera que existen suficientes fundamentos jurídicos para que todos los CAS, con cierto tiempo de servicios, ingresen sin concurso al régimen del servicio civil. No obstante, se está desarrollando esta propuesta, bajo la hipótesis de que tras una posible declaración de inconstitucional de la Ley $n .^{\circ} 31131$, se determine el retorno de la temporalidad en el contrato administrativo de servicios, escenario donde los trabajadores presenciales de los hospitales requieren medidas de urgencia.
} 
Se advierte claramente que la propuesta comparte varias similitudes con las leyes mencionadas, pero a diferencia de la Ley $n .^{\circ} 31131$ se dirige a un sector específico de servidores públicos: los trabajadores de los hospitales que han estado prestando servicios presenciales durante el estado de emergencia sanitaria; $y$, que han accedido a su puesto de trabajo mediante concurso público.

Por otro lado, quedan excluidos aquellos que fueron contratados en el marco de la emergencia sanitaria, exceptuados de los alcances del artículo 8 del Decreto Legislativo n. ${ }^{\circ} 1057$, que dispone el concurso público como requisito de ingreso al régimen de la contratación administrativa de servicios.

Finalmente, si se aprobara una propuesta con estos fines, sería necesario indicar que la misma no se aplicará sobre los servidores que se encuentran en un proceso de nombramiento que concluirá dentro del primer año de su vigencia, a consecuencia de la aplicación de otras normas; por ejemplo: la Ley $\quad n .^{\circ} 31059$, que autoriza el nombramiento progresivo como mínimo del veinte por ciento (20\%) de los profesionales de la salud, técnicos y auxiliares asistenciales de la salud que a la entrada en vigencia del Decreto Legislativo n. ${ }^{\circ} 1153$ tuvieron vínculo laboral y fueron identificados en el marco de la Disposición Complementaria Final Nonagésima Octava de la Ley n. ${ }^{\circ} 30693^{27}$.

\section{Evaluación de la propuesta normativa}

Consolidar las medidas de protección señaladas requiere del máximo respeto al orden jurídico vigente. Ese es el problema de la Ley $n .^{\circ} 31131$, la cual consolida la existencia de regímenes diferenciados (Decretos Legislativos $n .^{\circ} 276$ y 728, entre otros regímenes), que afectan el derecho a la igualdad, señalado en el art. 2, 2 de la Constitución Política del Perú (CPP); contravienen la implementación del servicio civil o la carrera pública, que es un bien constitucional, señalado en el art. 40 de la CPP; afecta el equilibrio presupuestal, señalado en el art. 78 de la misma norma; entre otros ${ }^{28}$ y todo ello sobre la base de que existe un régimen de contratación laboral que resulta lesivo para los servidores públicos y debe derogarse. Aunque dicho fundamento sea real, no podrá soportar el desarrollo de medidas que atenten contra el orden jurídico, es decir: la incorporación de los servidores en dos regímenes distintos, cuando desde hace años el Poder Ejecutivo viene implementando un régimen único para los servidores del Estado, lo que consolida las diferencias que tanto se critican en las entidades.

En cambio, abordar el problema aplicando un tratamiento diferenciado, podría resguardar a este grupo de trabajadores. El artículo 103 de la Constitución Política el Perú señala que pueden expedirse leyes especiales de acuerdo con la naturaleza de las cosas, pero no por las diferencias de las personas. Naturaleza de las cosas y diferencias de las personas son dos

\footnotetext{
27 "NONAGÉSIMA OCTAVA. Dispónese la conformación de una Comisión Sectorial, la misma que se sujeta a lo establecido por la Ley 29158 , Ley Orgánica del Poder Ejecutivo, encargada de revisar los últimos procesos de nombramiento del personal de salud con la finalidad de identificar a los trabajadores que a la entrada en vigencia del Decreto Legislativo 1153 tuvieron vínculo laboral, cumplieron con las condiciones y requisitos establecidos por el Ministerio de Salud para el mencionado nombramiento, y no fueron incluidos en dichos procesos de nombramiento, así como de proponer al Poder Ejecutivo, en un plazo de hasta noventa (90) días calendario, el marco legal que corresponda para la solución integral del personal de salud no considerado en los mencionados procesos de nombramiento.

Las medidas que proponga la citada comisión se financian con cargo al presupuesto institucional del Ministerio de Salud, sin demandar recursos adicionales al Tesoro Público". De acuerdo con una nota aclarativa en el Sistema Peruano de Información Jurídica (SPIJ), con el Artículo 1 de la Ley $N^{\circ} 30957, "(. .$.$) se dispone que mediante la citada Ley se regula el nombramiento progresivo, en el año fiscal 2019, como mínimo del veinte por$ ciento (20\%) de los profesionales de la salud, técnicos y auxiliares asistenciales de la salud del Ministerio de Salud, sus organismos públicos y los gobiernos regionales y las comunidades locales de administración en salud (CLAS), que a la entrada en vigencia del Decreto Legislativo 1153 tuvieron vínculo laboral, cumplieron con las condiciones y requisitos establecidos por el Ministerio de Salud para el mencionado nombramiento, no fueron incluidos en los procesos de nombramiento correspondientes a los años 2014 al 2018 y fueron identificados en el marco de la presente disposición $(\ldots)^{\prime \prime}$.

${ }^{28}$ Para una visión más detenida de las crítiCas, se puede revisar Aguinaga, J. (2021). Desarrollo de las recientes normas sobre la contratación de personal bajo el régimen CAS a contratación de personal bajo el régimen CAS ¿Aparente disputa normativa? Soluciones Laborales, (160), 67-79.
} 
términos que sirven de filtro para determinar la constitucionalidad o inconstitucionalidad de una norma. El primero resulta relevante para que toda disposición se mantenga vigente en el ordenamiento jurídico y supere cualquier examen de validez. La naturaleza de las cosas hace referencia a una serie de circunstancias o condiciones que harían legítimo un tratamiento diferenciado. El porcentaje adicional que se brinda a las personas con discapacidad en los concursos públicos (Decreto Ley n. ${ }^{\circ} 29973$ ), la inclusión de los días de licencia por maternidad en el cómputo de las utilidades (Ley n. ${ }^{\circ}$ 30792), los beneficios económicos de los profesionales calificados que se desempeñan en el Fondo de Apoyo Gerencial (D. Ley n. ${ }^{\circ}$ 25650). Ayuda también, el hecho de que no se desconoce el régimen del servicio civil, lo que mantiene la propuesta dentro del marco jurídico vigente.

No obstante, el tratamiento diferenciado que se ha expuesto, como es lógico, podría motivar una serie de críticas basadas en la afectación del principio de igualdad, en tanto se permite ingresar a regímenes estables sin concurso público y tener una estabilidad en el CAS, que otros grupos de trabajadores no tienen. En este caso será necesario utilizar el test proporcionalidad29, que, en palabras de Javier Barnes (Burga, 2011), "(...) impide que se sacrifique inútilmente, más allá de lo necesario o en forma desequilibrada un derecho a favor de otro". Tomando en cuenta lo desarrollado en la primera parte del presente trabajo, este test sirve también para evaluar que la interacción entre diferencia e igualdad se ajuste lo máximo posible al Derecho, es decir: que las distinciones jurídicas sean legítimas.

Como se indicó en los párrafos finales del punto anterior, el test de proporcionalidad es la herramienta para revisar el ajuste constitucional de una norma que establece diferencias. El Tribunal Constitucional, en la sentencia de expediente n. ${ }^{\circ}$ 045-2004-AI/TC, desarrolla los pasos de este examen, los deben ser aprobados en su totalidad, caso contrario las medidas que se analizan serán inconstitucionales. A continuación, se hará una breve presentación de los mismos, teniendo en cuenta las consideraciones de la sentencia citada:

a) Determinación de la intervención en el principio derecho a la igualdad Implementar un trato distinto a los sujetos de aplicación, con la finalidad de obtener un fin que, preliminarmente podría verse opuesto a las reglas que prohíben discriminar.

b) Determinación de la intensidad de la intervención

El trato diferenciado puede surtir una serie de afectaciones que se pueden ser:

- Graves: si se desarrolla por alguna causa que la CPP rechaza; y, por resultado, impiden la práctica o disfrute de derechos fundamentales o constitucionales.

Medio: si se respalda por alguna causa proscrita por la CCP y como consecuencia impide la práctica o disfrute de derechos fundamentales o constitucionales.

- Leve: si se respalda en causas que no son las que proscribe la CPP; y, producen como resultado impedir o afectar la práctica o disfrute de un derecho de rango legal o un interés legítimo.

c) Determinación de la finalidad del tratamiento diferente

La finalidad del tratamiento está compuesta por un objetivo y un fin. El primero es la situación jurídica que se quiere obtener con la implementación de una regulación diferenciada. El segundo es el bien que se realiza con la conformación del objetivo.

d) Examen de idoneidad del tratamiento diferente

\footnotetext{
${ }^{29}$ Tomando las investigaciones de Rubio (2011), nuestro Tribunal Constitucional proporcionó a la herramienta los siguientes nombres: i) Test de Razonabilidad en la sentencia del 18 de enero del 2010, expediente número 1473-2009-PA-TC, fj. 43; ii) Test de proporcionalidad; en la sentencia del 1 de febrero del 2010, expediente número 003-2008-PI-TC, fj. 52; iii) Test de igualdad, sentencia del 29 de marzo del 2006, expediente número 003-2008-PI-TC, fj. 128. Además reconoció los tres nombres juntos en la sentencia del 1 de febrero del 2012, expediente número 003-2008-PI-TC, fj. 52: Sobre la base del test de razonabilidad o proporcionalidad [STC 0027-2006-PI-TC], este colegiado considera pertinente aplicar el test de igualdad [los negreados son nuestros].
} 
En este tramo se analiza una relación entre el medio y el fin. Como se evalúa el establecimiento de diferencias, el análisis debe determinar si se consigue un fin constitucional con las distinciones efectuadas.

\section{e) Examen de necesidad del tratamiento diferente}

En este tramo del test se estudia si pudo implementarse medidas nada, o menos, gravosas para el derecho a la igualdad. Para ello se hará una comparación entre la medida desarrollada y los medios hipotéticos alternativos, que podrían ser menos lesivos y, como mínimo, igualmente efectivos.

f) Proporcionalidad en sentido estricto del tratamiento diferente

Superar este paso, define la constitucionalidad de la medida. Por el examen de proporcionalidad se verifica una relación proporcionalidad entre la intensidad de la afectación y el grado de realización del objetivo de la medida empleada. Si la intensidad del cumplimiento de la finalidad constitucional es mayor a la afectación, este examen queda superado.

Desarrollado lo anterior, corresponde evaluar la constitucionalidad de la propuesta presentada desde el test de proporcionalidad, cuyos pasos se han referido previamente.

\section{a) Determinación de la intervención en el principio derecho a la igualdad:}

Se propone la incorporación de los servidores con Cas que prestan servicios presenciales en los hospitales a los regímenes laborales que les corresponda, según el centro de trabajo. Posteriormente, sobre el mismo grupo de profesionales, se propone su incorporación, sin concurso, en el régimen del servicio civil, regulado por la Ley $n .^{\circ}$ 30057 y su Reglamento General aprobado por el Decreto Supremo n. ${ }^{\circ} 040-2014-P C M$. En todos los Casos la incorporación se realiza de manera directa, atendiendo a un tiempo mínimo de permanencia mayor al periodo de prueba. Es decir, no se someten a los servidores beneficiarios a los concursos públicos regidos por el principio mérito. Mientras dichas incorporaciones se realicen, las contrataciones administrativas de servicios de los servidores señalados tendrán la condición de indefinidas. No les resulta aplicable la extinción por vencimiento el plazo.

\section{b) Determinación de la finalidad del tratamiento diferente}

Se busca dar un reconocimiento al sacrificio del personal médico y asistencial que durante años se ha desempeñado mediante la contratación administrativa de servicios y que ahora, durante el periodo de crisis sanitaria, ha sido el grupo de profesionales que más expuesto se encuentran. Asimismo, se busca proteger la fuente de trabajo de estos profesionales, que luego del sacrificio de la primera ola, suscitada entre noviembre y diciembre del 2020, se vieron sujetos a despidos que se amparaban en la temporalidad del CAS, a pesar de que varios de ellos se han desempeñado por muchos años en sus respectivos centros de trabajo (hospitales).

No sería la primera vez que el ordenamiento jurídico brinda derechos en mérito al aporte que ha brindado un grupo de personas. En el Perú, existen normas que otorgan beneficios o derechos en función a la contribución que diversos ciudadanos han brindado a la nación. Por ejemplo, mediante resolución legislativa se puede brindar pensión de gracia, equivalente a dos remuneraciones mínimas, en atención a los aportes culturales que realicen. Asimismo, con la Ley del Veterano de Guerra y de la Pacificación Nacional, Ley $n .^{\circ} 30826$, se otorga una serie de derechos como el acceso gratuito a todos los museos, atractivos históricos y turísticos del Estado; lugares preferenciales para la Parada Militar y ceremonias públicas oficiales; promoción de beneficios económicos por parte de las empresas privadas. Asimismo, con la Ley $n .^{\circ}$ 
30655, que declara héroes de la democracia a los miembros del Grupo Especial de Inteligencia - Gein - Dircote - PNP, se reconoce a los miembros de este equipo, a sus hijos, cónyuge o concubina el acceso a becas y el otorgamiento de facilidades económicas que permitan continuar estudios de educación superior. De similar forma con la Ley n. ${ }^{\circ} 30554$, Ley que declara Héroes de la Democracia a los Comandos Chavín de Huántar.

Entonces, el Estado Peruano tiene la práctica de manifestar reconocimiento a los ciudadanos que han contribuido de manera significativa con la nación. Teniendo en cuenta el sacrificio constante que realiza el personal de los hospitales a nivel nacional, se explica la finalidad de la presente propuesta normativa.

\section{c) Determinación de la intensidad de la intervención}

Con esta a propuesta normativa se interviene el derecho a la igualdad, en tanto cierto grupo de profesionales o trabajadores se vería exceptuado de ingresar al público mediante concurso, cuyo componente definitorio es el mérito.

No obstante, debe atenderse que esta distinción no opera en función de un motivo proscrito. De lo que se trata aquí es de brindar un justo reconocimiento a la intervención de estos profesionales en la peor crisis sanitaria de nuestra nación, acción que el Estado tiene por costumbre realizar, dado el marco jurídico citado en el paso previo de este análisis.

Entonces, al tener la distinción propuesta cierta repercusión sobre un derecho fundamental, en base a motivos no proscritos por el ordenamiento jurídico, la intensidad de la intervención es leve.

\section{d) Examen de idoneidad del tratamiento que se propone}

En este trabajo se ha presentado una propuesta en términos generales; es decir, no se ha desarrollado un proyecto normativo, aunque por la finalidad del mismo debería ser una ley, la cual constituye una herramienta adecuada para el fin que se persigue.

Corresponde abordar, ahora, si el tratamiento que se propone es pertinente para lograr un efectivo reconocimiento de la labor brindada por el personal médico y asistencial de los hospitales del Ministerio de Salud que se desempeña mediante contrato administrativo de servicios, durante la crisis sanitaria originada por la Covid-19.

Es necesario tener en cuenta que el desarrollo de labores en el ambiente más peligroso para cualquiera de los trabajadores que se encuentra en la primera línea de atención representa la puesta en riesgo del bien más valioso que nuestra Constitución Política del Perú protege: la vida. En ese sentido, cualquier medida congratulativa que el Estado disponga no sería suficiente para compensar la puesta en riesgo de la propia existencia, de un grupo de profesionales que combaten una enfermedad que tiene en zozobra al país.

No obstante, la disparidad entre el valor del bien arriesgado y el valor de los beneficios que se proponen mediante la propuesta, es evidente que su otorgamiento es suficiente para consolidar un reconocimiento. Ello en tanto el régimen en el que se desenvuelven no les ofrece la garantía de un trato digno - no solo por una estabilidad laboral que podrían perderse en el marco de un pronunciamiento del Tribunal Constitucional, sino también porque carecen de la protección que otros regímenes otorgan. Ya se mencionó 
que, en los últimos momentos de la primera ola de la pandemia, a muchos de estos servidores se les remitió una carta de no renovación, a pesar de haber ingresado por concurso público y de tener muchos años de servicios - que no es prueba más que de una labor que se ha consolidado a base de mérito. Entonces, no hay mejor forma de reconocer el riesgo efectuado que emitir disposiciones que permitan a este personal tener una estabilidad, exceptuándolos de someterse nuevamente a un concurso público de méritos, mérito que ya lo han demostrado a lo largo de su carrera, sobre todo en este periodo de crisis sanitaria.

\section{e) Examen de necesidad del tratamiento diferente}

Dada la finalidad de la medida, ¿existen otras formas de reconocer la labor realizada por el por los trabajadores de salud (presenciales) de los hospitales a nivel nacional? Podría plantearse que, para brindar un reconocimiento merecido a estos trabajadores, bastaría con establecer beneficios similares a los que se les brindan a los héroes de guerra, o a los artistas, sin involucrar la incorporación directa en regímenes laborales de naturaleza permanente y sin involucrar el gasto estatal. No obstante, fueron condecoradas en base a beneficios que aún no poseían, por estar en un régimen laboral que no está sometido a la temporalidad que presenta la contratación administrativa de servicios. Por otro lado, podría formularse modificaciones benéficas que no implican la indeterminación del contrato CAS de estos trabajadores. No obstante, la finalidad de las propuestas es justamente dar un reconocimiento a su labor y evitar el escenario de no renovaciones abruptas del año 2020.

Entonces, cualquier otra medida que no implique asegurar laboralmente a estos trabajadores que han venido laborando en condiciones extremas - no sólo porque están en contacto directo con la enfermedad, sino también por las carencias del Estado en equipos de protección personal. Y dado que el régimen del Decreto Legislativo $n$. $^{\circ}$ 1057 está normativamente destinado a la extinción, la mejor forma de lograr esto es reconociendo la incorporación al régimen de sus centros de trabajo y, posteriormente, al régimen de la Ley $n .^{\circ} 30057$, de manera directa.

Es útil realizar un parangón con las resoluciones legislativas que disponen brindar una pensión equivalente a dos remuneraciones mínimas vitales las pensiones a los personajes de las ciencias o las artes. A las mismas se les paga en virtud de acciones con repercusión positiva a nivel nacional. Lo mismo sería en el caso de los servidores que prestan servicios en los hospitales, no obstante, ellos continuarán ejerciendo sus funciones.

\section{f) Examen de proporcionalidad en sentido estricto}

Considerando la sentencia del Expediente $n .^{\circ} 45-2004-P I$, mientras más se interviene el principio de igualdad, más debe cumplirse el fin constitucional de la medida.

Si bien es cierto, la aplicación de esta propuesta puede significar una intervención significativa en el principio de igualdad. Ello no significa que las personas cualificadas para postular al sector público se vean afectadas en tanto que la crisis sanitaria ha demostrado que existe una gran necesidad de profesionales de la salud que no se basta con el grupo de trabajadores que el presente proyecto de ley pretende beneficiar. En ese sentido, el Estado seguirá realizando convocatorias que desarrollarán en igualdad de condiciones entre los participantes de estas.

A diferencia de los efectos de la intervención señalada, el grado de cumplimiento de la finalidad de la propuesta es alto, en tanto se lograría brindar un reconocimiento 
adecuado a la puesta en riesgo de la vida del personal médico y asistencial; $y$, el mérito en el desempeño de sus funciones mediante su contratación administrativa de servicios.

\section{Conclusiones}

Igualdad y diferencia son dos elementos esenciales en el orden jurídico, que configuran el orden de la sociedad y el tratamiento de sus integrantes. Una regulación se ajusta a las particularidades de los sectores que conforman el orden social.

Los profesionales, técnicos, auxiliares y asistenciales de los hospitales a nivel nacional, son el grupo de trabajadores que más se exponen en la primera línea de contención de la Covid-19. Muchos de estos servidores, que ingresaron a sus centros de trabajo, previo concurso de méritos, con el régimen Cas han sido despedidos, o no renovados, entre noviembre y diciembre del 2020. Actualmente, gracias a la Ley n. 31131 - norma que surgió dentro de varias propuestas parlamentarias dirigidas a erradicar el régimen Cas, muchos de ellos tienen estabilidad, hasta que se efectúe el traslado al régimen laboral correspondiente. Dicha situación podría cambiar si es que el Tribunal Constitucional declara la Inconstitucionalidad de la norma; y, además, se dispone el retorno de la temporalidad de los contratos administrativos de servicios.

Ante dicha contingencia, se recomienda el establecimiento de un tratamiento diferenciado, por el que sus contratos CAS conserven la condición de indefinidos, en tanto se realiza su traslado al régimen de sus centros de trabajo; y, posteriormente al Régimen del Servicio Civil.

La propuesta podría recibir críticas basadas en la afectación del derecho a la igualdad. Sin embargo, tal como se expuso, esta logra superar el test de proporcionalidad. Debe tenerse en cuenta que las condiciones de riesgo son más elevadas en este grupo de trabajadores. Son mérito de sus acciones y el riesgo que implican las que justifican el establecimiento de estas medidas. Asimismo, debe recordarse que el Estado ha emitido normativa con objetivos similares.

\section{Bibliografía}

Aguinaga, J. (2021). Desarrollo de las recientes normas sobre la contratación de personal bajo el régimen CAS a contratación de personal bajo el régimen CAS ¿Aparente disputa normativa? Soluciones Laborales, (160), 67-79.

BERNALES BALLESTEROS, E (1999). La Constitución de 1993: análisis comparado, Quinta edición, Perú: RAO.

BOBBIO, N (1993). Igualdad y Libertad, España: Paidos.

BURGA CORONEL, A. M. (2011). El test de ponderación o proporcionalidad de los derechos fundamentales en la jurisprudencia del Tribunal Constitucional Peruano. Gaceta Constitucional (47), 253-267.

GUTIÉRREZ CAMACHO, W. Y SOSA SACIO, M (2005). Igualdad ante la Ley. En Gaceta Jurídica. La Constitución Comentada: Análisis artículo por artículo, Tomo I, Lima: Gaceta Jurídica. 
DEFENSORÍA DEL PUEBLO (2007). La Discriminación en el Perú: Problemática, normatividad y tareas pendientes. Lima: Defensoría del Pueblo.

LANDA ARROYO, C. (2017). Los Derechos Fundamentales. Lima: Editorial PUCP.

NEVES, J. (2014) Introducción al Derecho del Trabajo. Lima: Fondo Editorial PUCP.

RUBIO, M. (2011). El test de proporcionalidad en la jurisprudencia del Tribunal Constitucional peruano. Lima: Fondo Editorial Pontificia Universidad Católica del Perú.

\section{Editor}

Dra. Dora Ojeda Arriaran

\section{Cómo citar este trabajo}

Aguinaga Saavedra, J. (2021). El Contrato Administrativo de Servicios, los trabajadores de los hospitales y la eventual necesidad de establecer un tratamiento jurídico diferenciado, Vol. 10 (No. 1), 5-16 doi: https://doi.org/10.35383/ius-usat.v10i1.605

\section{Financiación}

El presente artículo no cuenta con financiación específica de agencias de financiamiento en los sectores público o privado para su desarrollo y/o publicación.

\section{Conflicto de interés}

El autor / Los autores del artículo declara(n) no tener ningún conflicto de intereses en su realización.

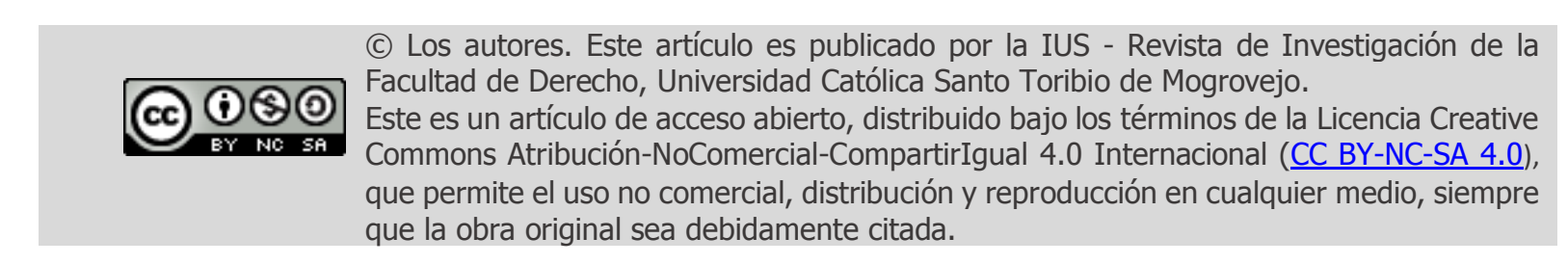

